Haben Sie Fragen zur Abrechnung oder zur wirtschaftlichen

\title{
Hausärzte, gründet MVZs!
}

? Dr. H. G., Allgemeinarzt, Bayern: Wir sind eine hausärztliche Gemeinschaftspraxis mit zwei Arztsitzen und einem angestellten Arzt auf einem weiteren Arztsitz. Die neue Gesetzgebung erlaubt jetzt auch fachgruppengleiche Medizinische Versorgungszentren (MVZ). Ergeben sich daraus Vorteile?

! MMW-Experte Walbert: Grundsätzlich ja. In einer Einzelpraxis oder Berufsausübungsgemeinschaft (BAG) bestehen wegen des Grundsatzes der persönlichen Leitung Einschränkungen: Auf einen voll zugelassenen Arztsitz können maximal drei weitere Ärzte angestellt werden, damit die „persönliche Leitung“ gewährleistet ist. In einem MVZ dürfen, sofern der Planungsbereich nicht gesperrt ist, Ärzte in unbe-

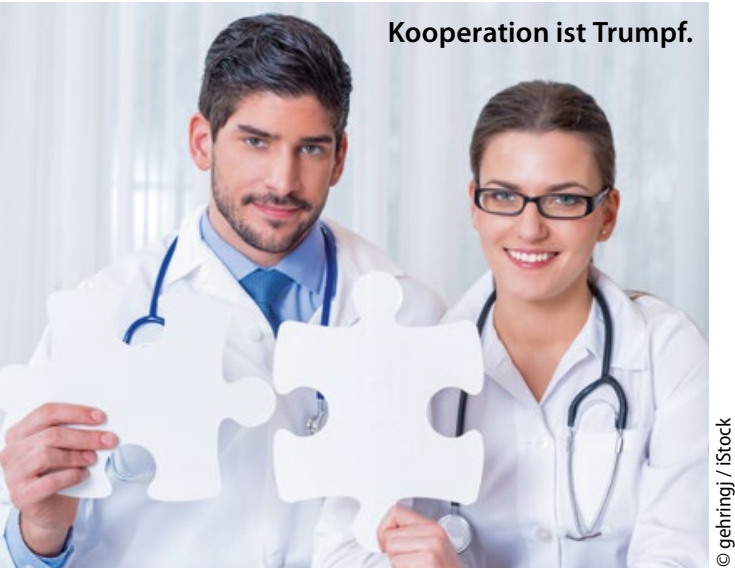

grenzter Zahl angestellt werden. Zudem kann ein MVZ mit angestellten Ärzten in nicht gesperrten Bereichen an mehreren Standorten tätig werden.

Auch aus unternehmerischer Sicht hat das MVZ einen erheblichen Vorteil: Es ist die Rechtsform einer Gesellschaft mit beschränkter Haftung $(\mathrm{GmbH}) \mathrm{zu}$ lässig. In einer BAG oder einer Personengesellschaft haften alle Ärzte außer den angestellten mit ihrem gesamten Vermögen. Dieses Risiko kann die $\mathrm{GmbH}$ beschränken.

Für junge Ärzte bietet ein MVZ mit der Freistellung von Organisation, unternehmerischen Entscheidungen und betriebswirtschaftlichen Überlegungen darüber hinaus einen attraktiven Arbeitsplatz, an dem noch dazu Teilzeitmodelle problemlos umgesetzt werden können. Als MVZ-Mitbetreiber findet man daher einfacher einen Nachfolger und kann danach im selbst gewählten Umfang weiterhin mitarbeiten.

In einem MVZ kann außerdem die Wettbewerbsfähigkeit erheblich gesteigert werden. Ein ausgedehntes Zeitleistungsangebot lässt sich hier viel leichter verwirklichen. Auch das ärztliche Leistungsangebot kann durch Spezialisierung und technische Ausstattung wirtschaftlich ausgebaut werden. Durch höhere Patientenzahlen lassen sich Investitionen in leistungsfähige Geräte leichter amortisieren und höhere Gewinne erwirtschaften.

\section{Arbeitsrecht}

\section{Keine Pflicht zu Überstunden}

\section{$?$ Dr. P. W., Hausarzt, Baden-Würt- temberg: Eine Mitarbeiterin hat sich geweigert, Überstunden zu ma- chen. Sind Arbeitnehmer nicht grund- sätzlich dazu verpflichtet? Liegt hier ein Grund zu Abmahnung vor?}

MMW-Experte Walbert: Grundsätzlich braucht kein Mitarbeiter Überstunden zu leisten. Es gibt allerdings wenige Ausnahmen:

1. Im Arbeitsvertrag, in Tarifverträgen oder Betriebsvereinbarungen sind Voraussetzungen für Überstunden bzw. Mehrarbeit vereinbart. Beispiel für den Arbeitsvertrag: „Der Arbeitnehmer ist verpflichtet, auch über die vertraglich vereinbarte Arbeitszeit hinaus auf Anforderung Überstunden zu leisten."

2. Es wird eine Vereinbarung im Einzelfall getroffen, mit der der Mitarbeiter einverstanden ist. Das geht auch mündlich, darf aber keiner Regelung im Tarifvertrag entgegenstehen.

3. In Notfällen dürfen Überstunden angeordnet werden, allerdings nur bei $\mathrm{Ge}$ fahren für den Betrieb oder unvorhersehbaren äußeren Ereignissen.

Liegen diese Ausnahmen nicht vor, können Überstunden verweigert werden. Es gibt keinen Grund für eine Abmahnung. Ein Tipp: Arbeitsverträge können natürlich im gegenseitigen Einvernehmen geändert werden. 